\title{
Correlative SEM and Raman imaging of hot spots on SERS substrate
}

\author{
R. Váňa ${ }^{1}$, M. Kocman ${ }^{1}$ and J. Jiruše ${ }^{1}$ \\ ${ }^{1}$ TESCAN Brno, s.r.o., Libusina trida 1, Brno, Czech Republic
}

Surface Enhanced Raman Spectroscopy (SERS) is becoming more popular due to large signal amplification of the Raman signal and therefore for a quick chemical analysis. The enhancement of the signal is caused by amplification of the light by the excitation of localized surface plasmon resonances. This light concentration occurs usually in the gaps, crevices, or sharp features of plasmonic materials, which contain metals with nanoscale features. The spots which enhance the field are called ,hot spots', and their size varies between a few and several hundred $\mathrm{nm}$. We were able to visualize hot spots using a recently developed correlative Scanning Electron (SEM) and Confocal Raman (CRM) microscopy [1] we call RISE. Visualization of exactly the same area of the SERS substrate in SEM and Raman is very challenging in two stand-alone systems since the plasmonic nanostructures on substrates are usually periodically arranged and similar to each other. The unique configuration of the RISE system lies in the direct navigation between SEM image and corresponding area for Raman spectra acquisition. Figure 1 shows overlaid SEM and Raman images of mercaptopyridine on silver SERS substrate with corresponding spectra [2]. Different enhancement factor of the Raman signal of mercaptopyridine can be seen due to variance of the plasmonic nanostructures on the periodically ordered, plasma etched, and silver-coated polystyrene spheres.

The RISE system comprises a high-resolution SEM and CRM integrated into the vacuum chamber. The CRM is equipped with a green excitation laser $(532 \mathrm{~nm})$ which provides a lateral resolution of $360 \mathrm{~nm}$ and depth resolution of $750 \mathrm{~nm}$, respectively. Thus the resolution of CRM is comparable with standalone Raman instruments and it is not compromised by integration with the SEM. The electron microscope used here comprises the immersion magnetic optics with a resolution of $1.4 \mathrm{~nm}$ at $1 \mathrm{kV}$ and $1 \mathrm{~nm}$ at $15 \mathrm{kV}$ [3], however, other types of electron columns are possible as well. The high resolution at low electron acceleration voltages makes it suitable for beam-sensitive samples.

\section{References:}

[1] J Jiruše et al, Journal of Vac. Sci. Technol. B 32 (2014), 06FC03.

[2] L Štolcová et al, Proceedings of Progr. in Elmag. Res. Symp. (PIERS), Stockholm (2013), 426.

[3] J Jiruše et al, Microsc. Microanal. 19 Suppl. 2 (2013), 1302.

[4] The research leading to these results has received funding from the European Union 7th Framework Program [FP7/2007-2013] under grant agreement n²80566, project UnivSEM. 

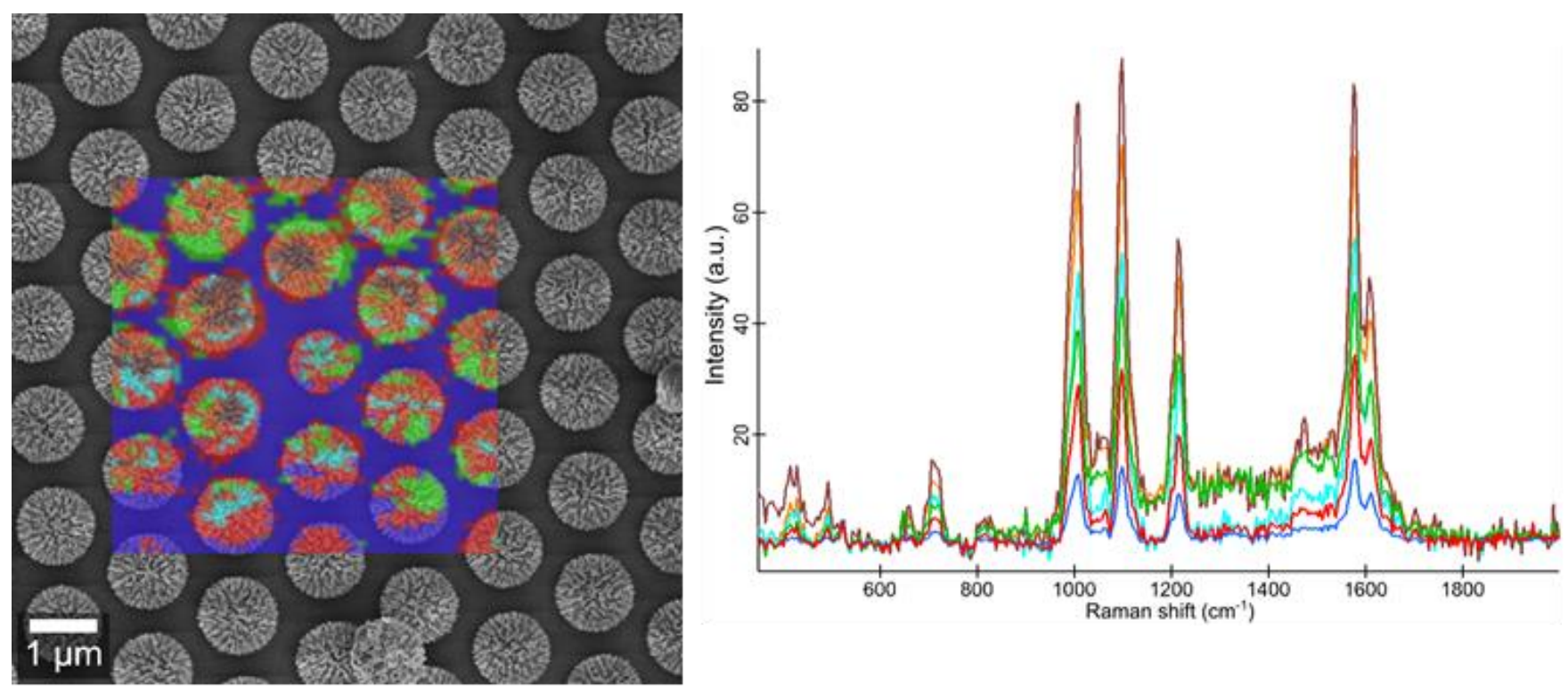

Figure 1: A detailed view of SERS substrate with correlative SEM-Raman imaging. Left: Overlaid SEM and Raman micrographs, Right: Corresponding Raman spectra showing characteristic peaks of mercaptopyridine. Various colors correspond to the different enhancement factor of the spectra caused by variance of the plasmonic structures on polystyrene spheres. 